Check for updates

Cite this: RSC Adv., 2018, 8, 7450

\title{
In situ synthesis and electronic transport of the carbon-coated AgaC/MWCNT nanocomposite
}

\author{
Dongxing Wang, ${ }^{a}$ Da Li, ${ }^{b}$ Javid Muhammad, ${ }^{a}$ Yuanliang Zhou, (D) a Ziming Wang, ${ }^{a}$ \\ Sansan Lu, ${ }^{a}$ Xinglong Dong (D) ${ }^{* a}$ and Zhidong Zhang ${ }^{* b}$
}

A nanocomposite of AgaC nanocapsules dispersed in a multi-walled carbon nanotube (MWCNT) matrix was fabricated in situ by a facile arc-discharge plasma approach, using bulk $\mathrm{Ag}$ as the raw target and methane gas as the carbon source. It was found that the AgaC nanocapsules were $\sim 10 \mathrm{~nm}$ in mean diameter, and the MWCNTs had 17-32 graphite layers in the wall with a thickness of 7-10 nm, while a small quantity of spherical carbon cages (giant fullerenes) were also involved with approximately 2030 layers of the graphite shell. Typical dielectric behavior was dominant in the electronic transport of Ag@C/MWCNT nanocomposites; however, this was greatly modified by metallic Ag cores with respect to pure MWCNTs. A temperature-dependent resistance and $I-V$ relationship provided evidence of a transition from Mott-David variable range hopping $\left[\ln \rho(T) \sim T^{-1 / 4}\right]$ to Shklovskii-Efros variable range hopping $\left[\ln \rho(T) \sim T^{-1 / 2}\right]$ at $5.4 \mathrm{~K}$. A Coulomb gap, $\Delta_{\mathrm{C}} \approx 0.05 \mathrm{meV}$, was obtained for the AgaC/MWCNT nanocomposite system.

Received 4th January 2018

DOI: $10.1039 / \mathrm{c} 8 \mathrm{ra00078f}$

rsc.li/rsc-advances

fullerene family has been extensively investigated and show fundamental importance in the advancement of science and engineering as a consequence of their unique electronic and magnetic properties. ${ }^{12,13}$ To meet increasingly high requirements for applications, the fabrication of composites through the integration of CNTs with complementary metal nanoparticles has proved an effective route to gain excellent performances. $^{\mathbf{1 4}}$

Silver is a typical metal with a low melting point, high electrical conductivity, electrochemical activity, and favorable specialties. In recent years, nanocomposites of Ag nanoparticles combined with carbon nanotubes (Ag/CNTs) have been synthesized and applied in many fields. For instance, Melvin et al. evaluated the electromagnetic wave absorption properties of single/double-layer Ag/CNTs nanocomposites. ${ }^{15}$ Ma et al. reported highly conductive flexible adhesives (CFAs) composed of nanoscale Ag flakes, CNTs and nitrile butadiene rubber. ${ }^{16}$ Pillai et al. reported a conductive hybrid network consisted of silver nanowires (Ag NWs) and SWCNT, which has been demonstrated potential in high energy density flexible-solid-state supercapacitor. ${ }^{17}$ Dong et al. fabricated the Ag/CNTs networks for a highly conductive film. ${ }^{18} \mathrm{Li}$ et al. theoretically investigated the atomic and electronic structures of silver-filled SWCNTs by using first-principles calculations. ${ }^{19}$ Feng et al. revealed that the electrical resistivity of Ag/CNT composite increases slightly with the volume fraction of CNTs in a volume range below $10 \mathrm{vol} \%{ }^{20}$ Luo et al. synthesized Ag/CNT sorbent for the removal of elemental mercury from flue gases. ${ }^{21}$ Jung et al. also prepared airborne $\mathrm{Ag} / \mathrm{CNT}$ hybrid nanoparticles, for use as the antimicrobial in air filtration. ${ }^{22}$ In the above studies, almost all Ag/CNT

\footnotetext{
${ }^{a}$ Key Laboratory of Materials Modification by Laser, Ion and Electron Beams (Ministry of Education), School of Materials Science and Engineering, Dalian University of Technology, Dalian 116023, China. E-mail: dongxl@dlut.edu.cn; Tel: +86-411-84706130

${ }^{b}$ Shenyang National Laboratory for Materials Science, Institute of Metal Research, International Center for Materials Physics, Chinese Academy of Sciences, 72 Wenhua Road, Shenyang 110016, People's Republic of China. E-mail: zdzhang@imr.ac.cn; Tel: $+86-24-23971859$
} 
composite systems were fabricated by mechanically mixing CNT powders and $\mathrm{Ag}$ nanoparticles in solvents, while a few were obtained via an aerosol approach or a chemical reaction method. ${ }^{22,23}$ The most interesting research has concerned the electrical properties of Ag/CNT nanocomposites, which depend on the intrinsic capabilities of the individual parent, and also on their morphologies and interfacial characteristics. However, the electronic transport behaviors have rarely been examined to date.

To date, the fabrication of Ag/CNT nanocomposites has been achieved through a large number of strategies. To the best of our knowledge, the process of arc-discharge plasma has not been reported for the synthesis of such multicomponent composites. Here, this facile method was first applied in a onestep synthesis of the composite with carbon-coated $\mathrm{Ag}$ nanocapsules (Ag@C) embedded in a MWCNT matrix. Arc-discharge plasma is a conventional method with the merits of easy operation, controllable preparation conditions, large yield of nanopowders, and less pollution in the environment. Investigation of the electronic transport behaviors of Ag@C/MWCNT composites were also carried out, which helps to gain an insight into the influence of $\mathrm{Ag} @ \mathrm{C}$ nanocapsules on the electronic performance of the assembled MWCNTs. A testing sheet for as-prepared Ag@C/MWCNT powders was prepared using a mechanical press without any binder, to insure its high purity and well-defined junctions among the nanoparticles and MWCNTs. Electronic transport performances have been measured in the temperature range from $2 \mathrm{~K}$ to room temperature.

\section{Experimental details}

\section{Synthesis of Ag@C/MWCNT nanocomposite}

Ag@C/MWCNT nanocomposite powders were prepared using arc-discharge plasma, as reported in our previous publication (Dong et al.). ${ }^{24}$ The raw target was pure bulk Ag $(99.99 \%$ in purity), which served as the anode for arc-discharge to be evaporated. A carbon rod was used as the opposite cathode. After evacuating the chamber to $1.0 \times 10^{-2} \mathrm{~Pa}$, it was filled with methane gas to $1.0 \times 10^{-2} \mathrm{MPa}$. After ignition of the arc, the voltage was maintained at about $20 \mathrm{~V}$, depending on the gap between the two electrodes, and the current was set at $100 \mathrm{~A}$. Following a series of manipulations (i.e., evaporation of $\mathrm{Ag}$ target, nucleation and growth of fine clusters and passivation for over 12 hours), the powder product was collected from the water-cooled wall of the work chamber.

\section{Structural characterization and resistivity measurement}

Crystal phases of as-prepared Ag@C/MWCNT powders were confirmed by X-ray diffraction (XRD; PANalytical Empyrean) using $\mathrm{Cu} \mathrm{K} \alpha$ radiation $(\lambda=1.5416 \AA)$. Morphologies, interfaces and crystallographic details were characterized by scanning transmission electron microscopy (STEM; NOVA NanoSEM 450, $300 \mathrm{keV}$ ). Raman spectra were recorded using a laser excitation wavelength of $632.8 \mathrm{~nm}$. X-ray photoelectron spectroscopy (XPS; Thermo Escalab 250Xi) utilizing monochromatic $\mathrm{Al} \mathrm{K} \alpha$ $(h v=1486.6 \mathrm{eV})$ radiation as the theta probe was adopted to analyze the surface species. For the resistivity measurements, the testing sample was prepared by pressing the as-prepared nanopowders into a slice under an axial pressure of $20 \mathrm{MPa}$ in a steel die, and cutting to a size of $10 \times 10 \mathrm{~mm}$. The temperature dependence of resistivity and the $I-V$ curve were measured on a physical property measurement system (PPMS; Quantum Design).

\section{Results and discussion}

\section{Structure, morphology and surface species of Ag@C/MWCNTs}

Fig. 1 shows the XRD pattern of the as-prepared Ag@C/MWCNT composite powder. The characteristic peaks at $2 \theta=38.0^{\circ}, 44.2^{\circ}$, $64.4^{\circ}$, and $77.3^{\circ}$ are the diffractions from the crystallographic planes (111), (200), (220), and (311) of the metallic Ag phase (PDF no. 00-004-0783), while the peak at $2 \theta=26.1^{\circ}$ corresponds to the (002) plane of the graphite phase (PDF no. 00-001-0646). The detected graphite phase was thought to be from the MWCNTs and onion carbon structures existing in the powder; both will be further confirmed by the following high-resolution transmission electron microscopy (HRTEM) images (see Fig. 2). No silver oxide is visible in the XRD pattern and XPS measurement (see Fig. 3), for the reason that the metal Ag particles are protected by carbonaceous layers on the surface. It is the carbon-coated Ag@C nanocapsules (NCs) that are present.

The morphologies and structures of Ag@C/MWCNT powders are observed as shown in HRTEM images of Fig. 2(a)-(f) under different magnifications. Fig. 2(a) and (b) show the full view of Ag@C/MWCNT powders; spherical Ag@C NCs, onedimensional MWCNTs and some carbon cages are found. To further confirm the composition of the powder, the energy dispersive X-ray (EDX) spectrum was recorded, as shown in Fig. 2(c). It is seen that the Ag@C/MWCNT composite consists of $\mathrm{Ag}(31 \mathrm{wt} \%)$ and $\mathrm{C}(69 \mathrm{wt} \%)$ elements, while a small quantity of carbon and the $\mathrm{Cu}$ element come from the sample holder

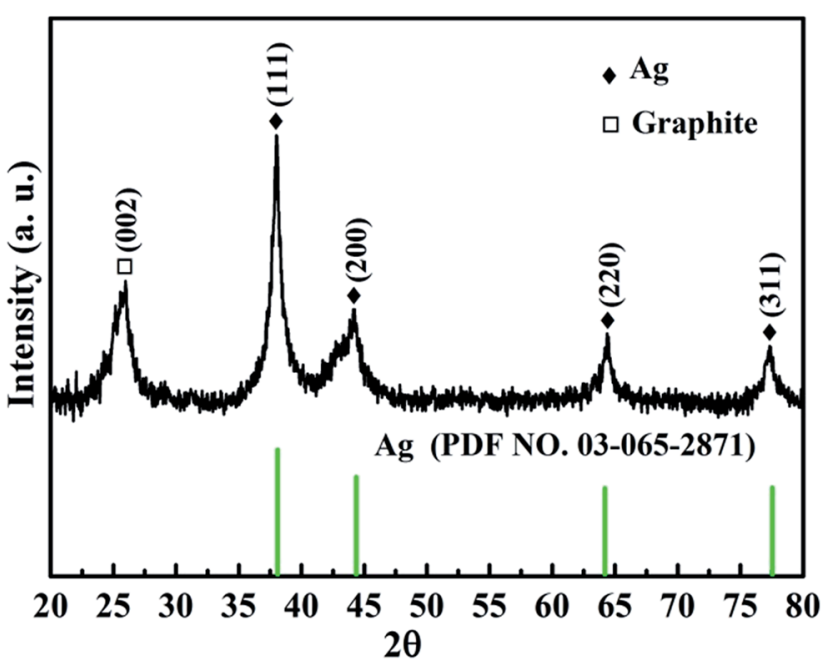

Fig. 1 XRD pattern of the as-prepared Ag@C/MWCNT composite powder. 

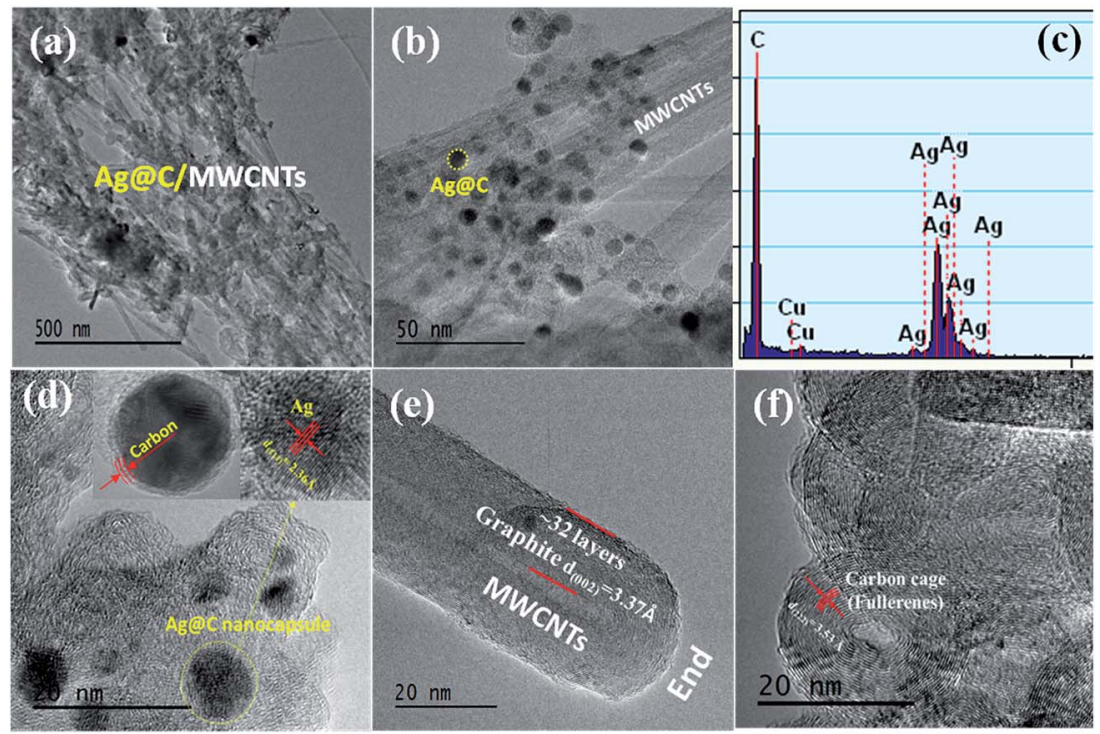

Fig. 2 HRTEM images and EDX spectrum of Ag@C/MWCNT composite powder. (a, b) Full views; (c) EDX spectrum; (d) Ag@C nanocapsule; (e) typical MWCNT; and (f) hollow carbon cages with an onion-like structure.
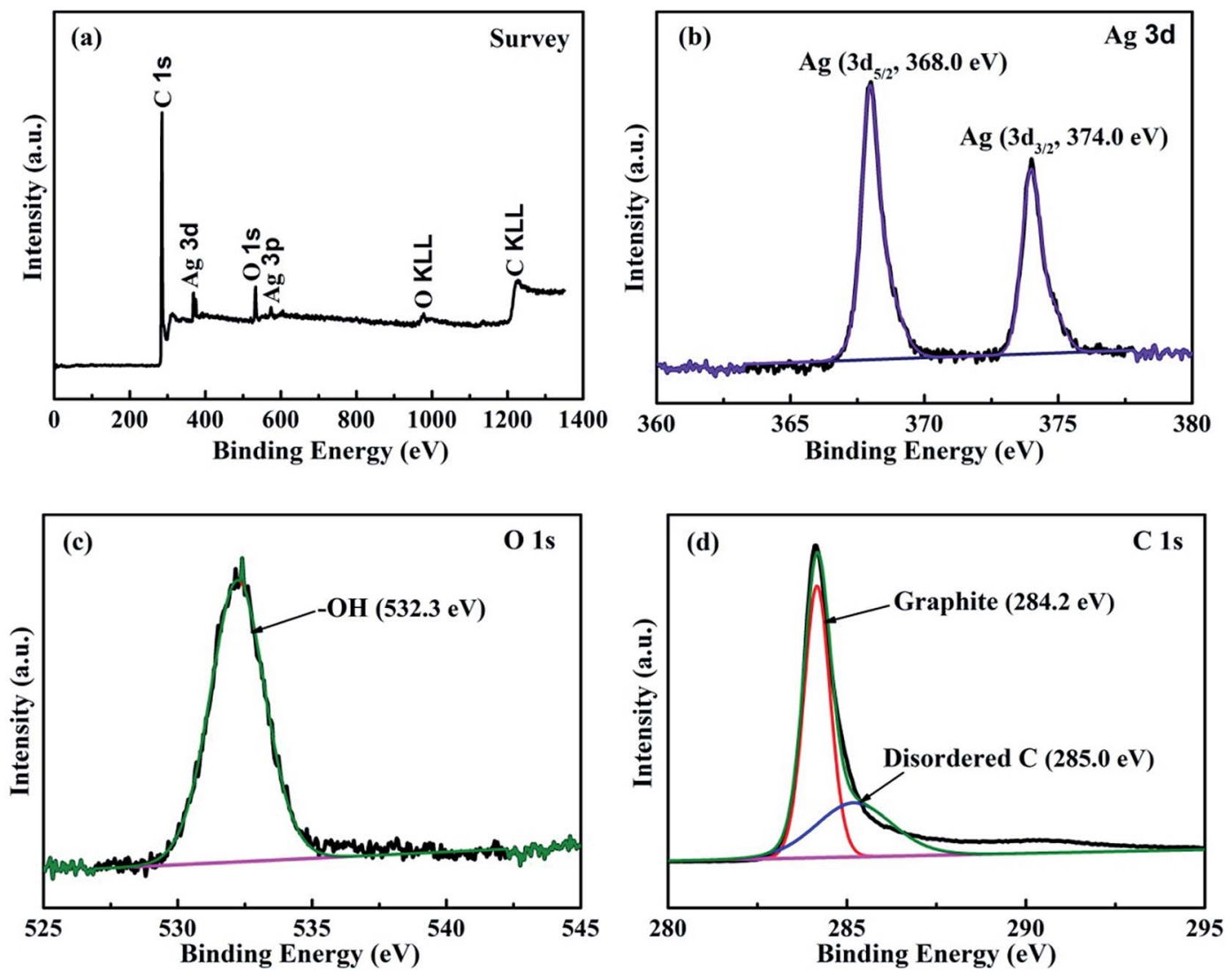

Fig. 3 XPS spectra of AgaC/MWCNT composite powder. (a) Survey spectra; (b) binding energies of Ag 3d electrons; (c) binding energies of O 1s electrons; (d) binding energies of $C$ 1s electrons.

used for the TEM observation. The mean size of the spherical $\mathrm{Ag} @ \mathrm{C} \mathrm{NCs}$ is $\sim 10 \mathrm{~nm}$ in diameter, and the $\mathrm{Ag}$ core is further confirmed by the detailed lattice image (inset of Fig. 2(d)), in which the single crystal $\mathrm{Ag}$ core is demonstrated by the lattice spacing of $0.236 \mathrm{~nm}$ matching to the distance of (111) plane in fcc-Ag. It is also seen in the inset of Fig. 2(d) that the Ag nanoparticle is covered with a disordered carbon shell, $2-3 \mathrm{~nm}$ in thickness, or embedded in the amorphous carbon matrix. 
Such carbon coating of Ag@C NCs has an effective ability to protect the active $\mathrm{Ag}$ core against oxidation, and is also the reason that no silver oxide is detected in the XRD pattern (Fig. 1) and XPS analysis (see Fig. 3(b)). A typical MWCNT, as displayed in Fig. 2(e), is mature, closed, and perfect in its structure, with $\sim 32$ graphite layers and an outside diameter of $\sim 30 \mathrm{~nm}$. It is obvious that MWCNTs have a highly oriented structure comprising of numerous parallel graphitic layers, retaining structural continuity even to the end of the CNT. As shown in Fig. 2(f), a large number of spherical carbon cages (giant fullerenes) are found with approximately 25-30 graphite layers in shells, verified from the interplanar spacing of $3.56 \AA$ belonging to (222) lattice planes of the fullerenes (PDF no. 00049-1717). The existence of hollow cages is considered to result from the migration of the metallic Ag core out of the original cage in the solidification process.

In order to identify the chemical composition of the Ag@C/ MWCNT composite powder, the XPS analysis was carried out as shown in Fig. 3. The survey spectra of Fig. 3(a) indicate the emissions from the $\mathrm{Ag}, \mathrm{C}$, and $\mathrm{O}$ elements, and the contents may be calculated as $6.61 \mathrm{wt} \%$ of $\mathrm{Ag}, 86.93 \mathrm{wt} \%$ of $\mathrm{C}$, and $6.41 \mathrm{wt} \%$ of $\mathrm{O}$. The oxygen is attributed to the adsorbed $-\mathrm{OH} / \mathrm{O}_{2}$ species in the air, the carbon arises from the MWCNTs, onion-like carbon cages, and disordered carbon nanostructures, while the Ag exists in the capsulated core-shell structures. Fig. 3(b)-(d) show the detailed photoelectron spectra of $\mathrm{Ag}$, O, and $\mathrm{C}$ elements, respectively. The peaks in Fig. 3(b) confirm pure Ag with zero valances through the binding energies of $\mathrm{Ag} 3 \mathrm{~d}$ electrons (368.0 eV for $3 \mathrm{~d}_{5 / 2}$, $374.0 \mathrm{eV}$ for $3 \mathrm{~d}_{3 / 2}$ electrons), ${ }^{25}$ without any silver oxides, which indicates that the pure $\mathrm{Ag}$ has been protected by the carbonaceous species. The feature peak of $\mathrm{O} 1$ s electrons at a binding energy of $532.26 \mathrm{eV}$ is attributed to the adsorbed $-\mathrm{OH} / \mathrm{O}_{2}$ species in air (Fig. 3(c)), while the absence of any peaks in the range $528-530 \mathrm{eV}$ (the binding energies for Ag-O) suggests no silver oxide passivated in the composite. Fig. 4(d) reveals the detailed spectrum of the $\mathrm{C}$ element, with the peak of $\mathrm{C} 1 \mathrm{~s}$ electrons at binding energies of 284.22 $\mathrm{eV}$ and $285.0 \mathrm{eV}$ corresponding to the $\mathrm{sp}^{2}$-hybridized

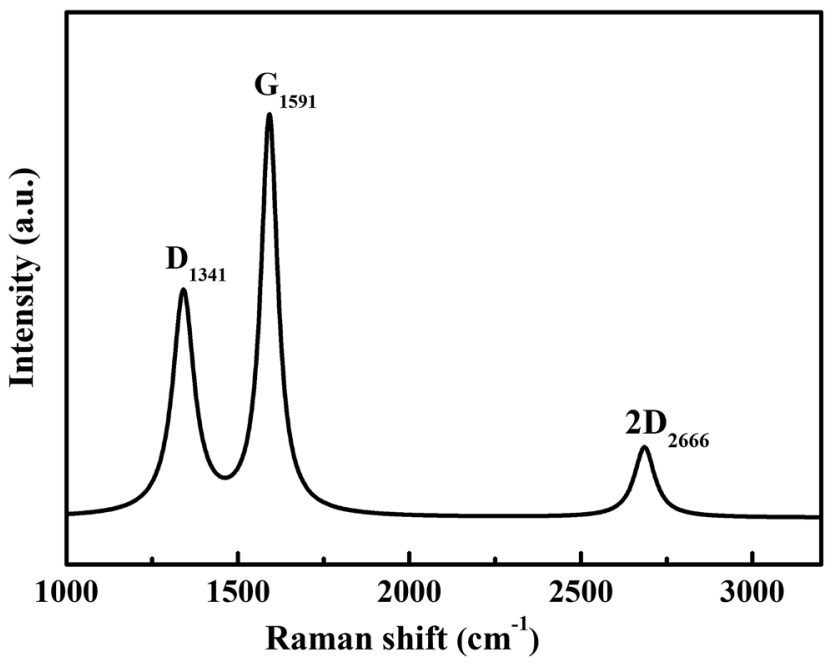

Fig. 4 Raman spectrum of the Ag@C/MWCNT composite powder. graphite-like carbon atoms of MWCNTs and the $\mathrm{sp}^{3}$-hybridized carbon atoms in a disordered structure, respectively. ${ }^{26}$

The graphitization degree of the Ag@C/MWCNT composite powder was analyzed by Raman spectra, as shown in Fig. 4. Two intensive peaks denoted as D-band at $1341 \mathrm{~cm}^{-1}$ and G-band at $1591 \mathrm{~cm}^{-1}$ correspond to the $\mathrm{A}_{1 \mathrm{~g}}$ and $\mathrm{E}_{2 \mathrm{~g}}$ of carbon vibration modes, respectively. The D-band suggests a disordered feature of the carbon structure and the G-band indicates perfect graphite. Both peaks are related to the $\mathrm{sp}^{2}$ electronic configuration containing electrons in the $\pi$ orbital and appear in the majority of Raman spectra of graphitic materials of a small crystallite size (so-called semicrystalline graphite). The D-peak, normally assigned to the presence of in-plane substitution heteroatoms, vacancies, grain boundaries, or other defects, indicates here a large amount of disordered carbon existing in the Ag@C/MWCNT powders, particularly in MWCNTs, onionlike carbon cages, and the disordered carbon structures, as observed in the HRTEM images of Fig. 2. A strong G-band is typical for graphite or carbon blacks, and originates from the stretching vibration of any $\mathrm{C}-\mathrm{C}$ pairs at $\mathrm{sp}^{2}$ sites, ${ }^{27}$ and represents here a high crystallinity and mature degree of MWCNTs and carbon cages. The intensity ratio of $I_{\mathrm{D}} / I_{\mathrm{G}}$ gives an intuition into the degree of carbonic crystallinity; here it is approximately 0.63 as obtained from a Lorentz function fitting on the Raman spectrum, which is smaller than 0.93 of pure MWCNTs. ${ }^{28}$ The value of $I_{\mathrm{D}} / I_{\mathrm{G}}$ suggests that the graphitization degree in the $\mathrm{Ag} @ \mathrm{C} / \mathrm{MWCNT}$ composite is relatively high, due to the high energy conditions of the arc-discharge plasma. Additionally, the peak denoted as 2D-band at around $2666 \mathrm{~cm}^{-1}$ is thought to originate from the finite crystal size of graphite, which is an overtone of the D-peak in the case of a total lack of $c$-axis order.

\section{Formation mechanism of one-step synthesized Ag@C/ MWCNT powder}

A schematic diagram for the in situ formation of Ag@C/MWCNT composite powder by arc-discharge plasma is shown in Fig. 5 . The plasma is a heat source which evaporates the bulk target into the gaseous state, then follows a series of nucleation, growth, diffusion, and passivation processes. The temperature of the arc flame may reach higher than $10000 \mathrm{~K}$, depending on the atmospheric conditions and electrical power supply. ${ }^{29}$ The gaseous carbon source $\left(\mathrm{CH}_{4}\right)$ and silver bulk are decomposed/ evaporated into energetic atoms/ions state (Fig. 5(a)), during which the ionized $\mathrm{H}^{+}$can further promote the evaporation of $\mathrm{Ag}$ bulk through an energy exchange, and work as small carrier atoms. The subsequent stage goes into nucleation of $\mathrm{Ag}$ seeds, which also induces the formation of MWCNTs according to a vapor-liquid-solid (VLS) mechanism ${ }^{30}$ (Fig. 5(b) and (c)). In VLS growth of CNTs, the second phase of the Ag particles, referred to as catalyst, directs and confines the growth of CNTs on a specific orientation and within a confined area. An Ag catalyst forms a liquid droplet by itself or by alloying with the growth material of the $\mathrm{C}$ atoms during growth, and also acts as a trap for carbonic species. Enriched growth species in the catalyst droplets subsequently precipitate at the growth surface, resulting in one-directional growth. It has been predicted that 


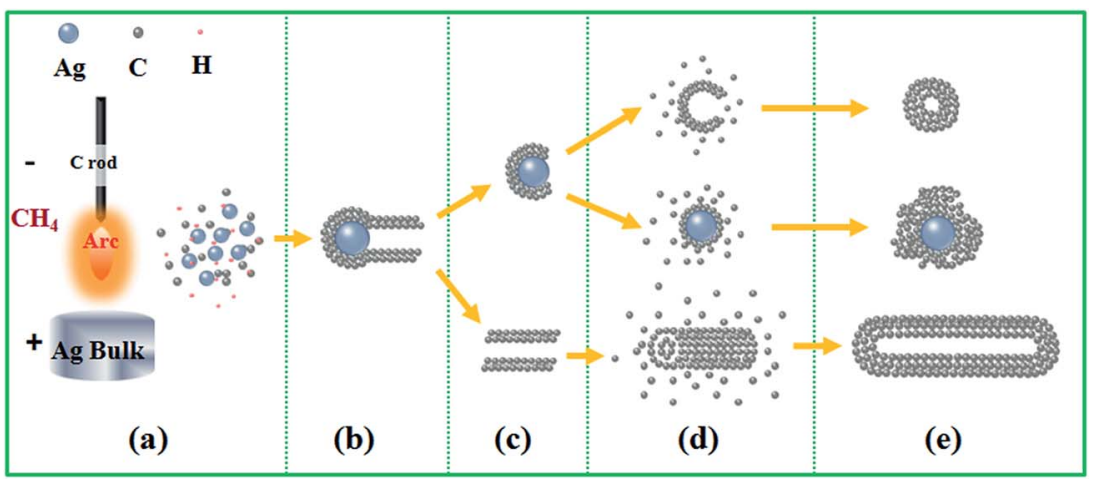

Fig. 5 Schematic formation of AgaC/MWCNT composite powder by in situ arc-discharge plasma. (a) Gasification of the Ag bulk; (b) absorption growth of $\mathrm{C}$ atoms; (c) separation of MWCNTs; (d) second absorption of C atoms; (e) formation of CNTs, Ag@C, carbon cages.

the $\mathrm{Ag}$ can serve as a catalyst to grow CNTs, due to the surface of Ag nanoparticles having the highest adatom chemical potential, which will absorb the $\mathrm{C}$ atoms from the saturated vapor. ${ }^{31}$ For the fcc metal crystallites (for example, the coinage metals of $\mathrm{Cu}$, $\mathrm{Ag}$, and $\mathrm{Au}$ ), it is confirmed that the (111) facets have the lowest energy; hence the lowest in adatom diffusion barriers, e.g. $0.20 \mathrm{eV}$ for the (111) facets of Ag. ${ }^{32}$ This implies that a significant acceleration of $\mathrm{C}$ atoms through diffusion can be achieved on specific facets of Ag particles, which favors the growth of CNTs along the $\langle 111\rangle$ direction and extends the $\mathrm{sp}^{2}$ carbon network involving hexagons and pentagons to form a crystallized graphite layer. The existence at the tip of MWCNTs or migration from the carbon cages make Ag particles separated from the well-defined carbon nanostructures (Fig. 5(d) and (e)). The isolated carbon cages can continuously grow by absorbing $\mathrm{C}$ atoms to form huge ones. The ultimately giant carbon fullerenes and the $\mathrm{Ag}$ nanoparticles are finally coated by thin layers of disordered carbon. It was reported earlier that the presence of Fe, Co, or $\mathrm{Ni}$ in the arc-discharge can stimulate the growth of the walled carbon tubes ${ }^{33,34}$ and strings of spherical beads. In this work, the same phenomena were also found; however, the carbon shell on the Ag nanocapsules was 1-2 $\mathrm{nm}$ in thickness, thinner than in the case of other metals.

\section{Electronic transport behaviors of Ag@C/MWCNT nanocomposite}

Fig. 6 shows the temperature-related electronic transport of the mechanically compressed Ag@C/MWCNT sheet. It is demonstrated in Fig. 6(a) that the dielectric behavior is dominant in the temperature range of 2-300 K, while the similar behavior to pure CNTs suggests that the MWCNTs are the main electric contributors, although the metallic Ag@C NCs are also involved in the composite..$^{35}$ The resistivity of the Ag@C/MWCNT nanocomposite increases with decreasing temperature, from 0.26 $\Omega \mathrm{cm}($ at $300 \mathrm{~K})$ to $2.43 \Omega \mathrm{cm}$ (at $2 \mathrm{~K})$, with a ratio $\rho_{\mathrm{r}}\left[\rho_{(2 \mathrm{~K})} / \rho_{(300 \mathrm{~K})}\right]$ of approximately 9.35. The temperature-dependent resistivity of carbonaceous composite is usually classified into three regimes: metallic, critical, and insulating regimes, depending on the extent of disorder. ${ }^{36}$ These regimes are divided from the plot of $\ln \rho$ vs. $\ln T$, as shown in Fig. 6(b), in which the transition temperature from metallic to insulating is determined as $24 \mathrm{~K}$. This means that the Ag@C/MWCNT composite will preserve its metallic features down to this low temperature. The important factors affecting the conductivity of carbonic species are the ratio of $\mathrm{sp}^{2}$ to $\mathrm{sp}^{3}$ and the clustering of the $\mathrm{sp}^{2}$ sites, which significantly control the electronic properties of the carbonbased materials. ${ }^{37}$ The status of $\mathrm{sp}^{2}$ to $\mathrm{sp}^{3}$ bonds in $\mathrm{Ag} @ \mathrm{C} /$ MWCNT nanocomposite is well presented in the Raman spectrum (see Fig. 5), indicated by the intensities of the D and G bands. Consequently, the electrons in Ag@C/MWCNT composite are strongly localized, and its conductivity is probably described by hopping conduction mode, which is controlled by the hopping of electrons between local states near the Fermi level. When the Coulomb interaction of electrons is negligible, a three-dimensional Mott-David (MD) VRH ${ }^{8}$ is expected for temperature-dependent resistivity:

$$
\begin{gathered}
\rho(T)=\rho_{0} \exp \left(\frac{T_{\mathrm{M}}}{T}\right)^{1 / 4} \\
T_{\mathrm{M}}=512 / 9 \pi \xi^{3} N\left(E_{\mathrm{F}}\right) k_{\mathrm{B}}
\end{gathered}
$$

where $T_{\mathrm{M}}$ is the characteristic Mott temperature obtainable from the plot of $\ln \rho(T) \sim T^{-1 / 4}, \xi$ is the localization length, $k_{\mathrm{B}}$ is the Boltzmann constant, and $N\left(E_{\mathrm{F}}\right)$ is the density of states at the Fermi level. A good linear fit is satisfactory with the experimental data, as represented in Fig. 6(c), indicating that the $\mathrm{Ag} @ \mathrm{C} / \mathrm{MWCNT}$ nanocomposite follows a three-dimensional MD VRH model in the temperature range from $5.4 \mathrm{~K}$ to $300 \mathrm{~K}$, with Mott temperature $T_{\mathrm{M}}=440 \mathrm{~K}$. Below $5.4 \mathrm{~K}$, a slight deviation appears from the fitting line, which implies a transition of the transport behavior occurred from MD to Shklovskii-Efros (SE) VRH mode. ${ }^{38}$ Details are presented in SE mode, as shown in Fig. 6(d). The Coulomb interaction between the electrons becomes significant in the SE mode, and the resistivity can be described by following relationship:

$$
\begin{gathered}
\rho(T)=\rho_{0} \exp \left(\frac{T_{\mathrm{SE}}}{T}\right)^{1 / 2} \\
T_{\mathrm{SE}}=2.8 e^{2} / \varepsilon \xi k_{\mathrm{B}}
\end{gathered}
$$



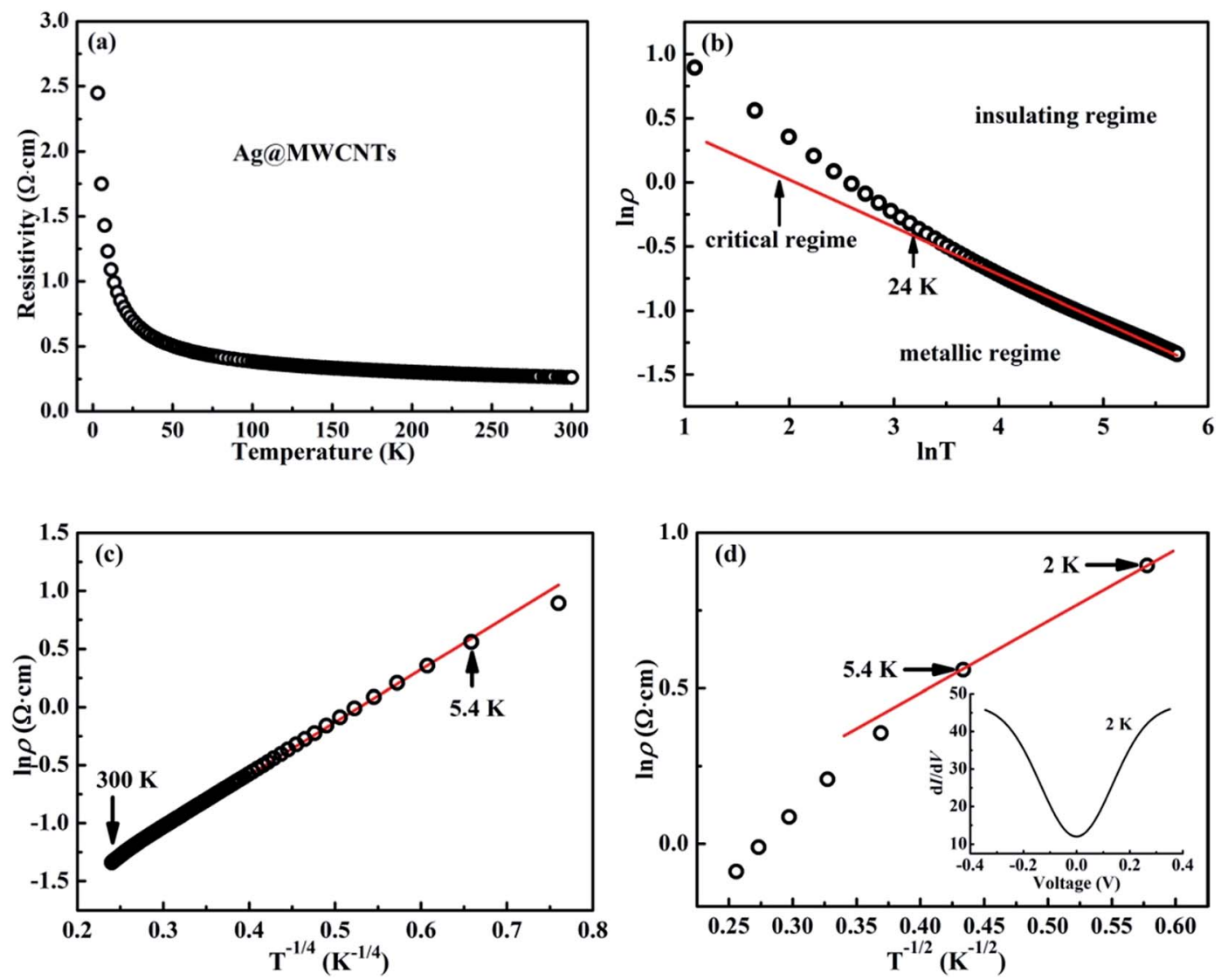

Fig. 6 (a) Temperature-dependent resistivity of the AgaC/MWCNT nanocomposite from 2 to $300 \mathrm{~K}$; (b) In-ln plot of the resistivity $\rho$ as a function of the temperature $T$ from 2 to $300 \mathrm{~K}$; (c) $\ln \rho \mathrm{Vs}$. $T^{-1 / 4}$ plot fitted with MD VRH mode, satisfactorily fitted in the temperature range of $5.4-300 \mathrm{~K}$; and (d) In $\rho$ vs. $T^{-1 / 2}$ plot fitted with SE VRH mode, further confirmed an applicable temperature range of $2-5.4 \mathrm{~K}$, and the inset shows plot of d// $\mathrm{d} V$ as a function of $V$ at $2 \mathrm{~K}$.

where $\rho_{0}$ is a material constant, $T_{\mathrm{SE}}$ is the characteristic SE temperature, and $\varepsilon=\varepsilon_{0}+4 \pi e^{2} \xi^{2} N\left(E_{\mathrm{F}}\right)$ is the dielectric constant. From the slope of the $\ln \rho(T) \sim T^{-1 / 2}$ line in Fig. 6(d), $T_{\mathrm{SE}}$ is calculated as $5.38 \mathrm{~K}$. Such a transition of the transport behavior from the MD to the SE mode has also been observed in CNTs or carbon fiber materials. ${ }^{35,39}$

It is well known that a Coulomb interaction between electrons plays an important role in the transport behavior of a disordered system. An obvious suppression in differential conductance at lower bias voltages is shown in the inset of Fig. 6(d). The depletion of the density of the states at low energies is always regarded as a signature of Coulomb interaction between electrons, in accordance with the gradual opening of a Coulomb gap at low temperatures. ${ }^{40}$ Efros and Shklovskii ${ }^{38}$ have proposed that the Coulomb gap $\Delta_{\mathrm{C}}$ can be obtained by the following relationship:

$$
\Delta_{\mathrm{C}}=e^{3} N\left(E_{\mathrm{F}}\right)^{1 / 2} / \varepsilon^{3 / 2}
$$

From eqn (2) and (4), we have

$$
\Delta_{\mathrm{C}} \approx 0.905 k_{\mathrm{B}} T_{\mathrm{M}}{ }^{-1 / 2} T_{\mathrm{SE}}{ }^{3 / 2}
$$

From here, a Coulomb gap $\Delta_{\mathrm{C}} \approx 0.05 \mathrm{meV}$ for the hybrid system is obtained. A transition point of electronic transport model has been reported at $15-16 \mathrm{~K}$ for the CNTs. ${ }^{35,41}$ Compared with above, the Ag@C/MWCNT composite has a much lower transition point, at $5.4 \mathrm{~K}$ (Fig. 6(d)). It might be reasonable that in the process of electron transport, silver nanoparticles can play the role of a bridge between the $\mathrm{C}-\mathrm{C}$; a large number of electrons can be free transport without being strongly localized. Therefore, the Coulomb interaction between electrons is weakened, so that the transition point of the transport model is backward delayed. It can be predicted that with increases in content of $\mathrm{Ag}$ nanoparticles, the model transition point will continue to be backward delayed, and eventually disappear.

\section{Conclusions}

Ag@C/MWCNT nanocomposite was prepared in situ by a modified arc-discharge plasma approach, through evaporation of bulk silver in a methane $\left(\mathrm{CH}_{4}\right)$ atmosphere. The Ag@C/MWCNT nanocomposite consists of carbon-coated Ag@C nanocapsules with a mean diameter of $\sim 10 \mathrm{~nm}$, MWCNTs with about 17-32 graphite layers in the wall and a thickness in the range of 7$10 \mathrm{~nm}$, and spherical carbon cages (giant fullerenes) with 
approximately 20-30 graphite layers in shell thickness. Temperature-dependent electrical resistivity reveals that the dielectric behavior is dominant in the Ag@C/MWCNT nanocomposite following MD VRH $\left[\ln \rho(T) \sim T^{-1 / 4}\right]$ mode and further transition into SE VRH $\left[\ln \rho(T) \sim T^{-1 / 2}\right]$ mode at $5.4 \mathrm{~K}$. Such a transition is thought to be due to an enhanced Coulomb interaction caused by the modification from metallic $\mathrm{Ag}$ cores dispersed in the MWCNT matrix. The Coulomb gap, $\Delta_{\mathrm{C}} \approx 0.05$ $\mathrm{meV}$, is obtained for this nanocomposite system. Such an in situ fabricated Ag@C/MWCNT nanocomposite is expected to be a promising semiconductor for various applications in electronic devices.

\section{Conflicts of interest}

There are no conflicts of interest to declare.

\section{Acknowledgements}

This work was financially supported by the National Natural Science Foundations of China (No. 51331006 and 51271044).

\section{References}

1 T. Chen and L. Dai, Mater. Today, 2013, 16, 272-280.

2 S. M. Jung, H. Y. Jung and J. S. Suh, Carbon, 2007, 45, 29172921.

3 M. Ding, Y. Tang, P. Gou, M. J. Reber and A. Star, Adv. Mater., 2011, 23, 536-540.

4 S. J. Tans, M. H. Devoret, H. Dai, A. Thess, R. E. Smalley, L. J. Geerligs and C. Dekker, Nature, 1997, 386, 474.

5 M. Bockrath, D. H. Cobden, J. Lu, A. G. Rinzler, R. E. Smalley, L. Balents and P. L. McEuen, Nature, 1999, 397, 598-601.

6 V. Krstic, S. Roth and M. Burghard, Phys. Rev. B: Condens. Matter Mater. Phys., 2000, 62, 16353-16355.

7 P. Poncharal, S. Frank, Z. L. Wang and W. A. D. Heer, Eur. Phys. J. D, 1999, 9, 77-79.

8 N. F. Mott and G. A. Davis, Electronic processes in noncrystalline materials, Clarendon, Oxford, 2nd edn, 1979.

9 T. C. L. G. Sollner, W. D. Goodhue, P. E. Tannenwald, C. D. Parker and D. D. Peck, Appl. Phys. Lett., 1983, 43, 588-590.

10 P. A. Lee, A. D. Stone and H. Fukuyama, Phys. Rev. B: Condens. Matter Mater. Phys., 1987, 35, 1039-1070.

11 R. A. Webb, S. Washburn, C. P. Umbach and R. B. Laibowitz, Phys. Rev. Lett., 1985, 54, 2696.

12 Y. F. Li, T. Kaneko and R. Hatakeyama, Appl. Phys. Lett., 2008, 92, 183115.

13 F. Lópezurías, E. Cruzsilva, E. Muñozsandoval, M. Terrones and H. Terrones, J. Mater. Chem., 2008, 18, 1535-1541.

14 Y. N. Xia, P. D. Yang, Y. G. Sun, Y. Y. Wu, B. Mayers, B. Gates, Y. D. Yin, F. Kim and H. Q. Yan, Adv. Mater., 2003, 34, 353389.

15 G. J. H. Melvin, Q. Q. Ni, T. Natsuki, Z. P. Wang, S. Morimoto, M. Fujishige, K. Takeuchi, Y. Hashimoto and M. Endo, Synth. Met., 2015, 209, 383-388.
16 R. Ma, S. Kwon, Q. Zheng, H. Y. Kwon, J. I. Kim, H. R. Choi and S. Baik, Adv. Mater., 2012, 24, 3344.

17 S. K. R. Pillai, J. Wang, Y. L. Wang, M. M. Sk, A. B. Prakoso, Rusli and M. B. Chan-Park, Sci. Rep., 2016, 6, 38453.

18 R. X. Dong, C. T. Liu, K. C. Huang, W. Y. Chiu, K. C. Ho and J. J. Lin, ACS Appl. Mater. Interfaces, 2012, 4, 1449.

19 W. F. Li, M. W. Zhao, Y. Y. Xia, T. He, C. Song, X. H. Lin, X. D. Liu and L. M. Mei, Phys. Rev. B: Condens. Matter Mater. Phys., 2006, 74, 195421.

20 Y. Feng, H. L. Yuan and M. Zhang, Mater. Charact., 2005, 55, 211.

21 G. Q. Luo, H. Yao, M. H. Xu, X. W. Cui, W. X. Chen, R. Gupta and Z. H. Xu, Energy Fuels, 2010, 24, 419.

22 J. H. Jung, G. B. Hwang, J. E. Lee and G. N. Bae, Langmuir, 2011, 27, 10256.

23 Z. Q. Tan, H. Xu, H. Abe, M. Naito and S. Ohara, J. Nanosci. Nanotechnol., 2010, 10, 3978.

24 X. L. Dong, Z. D. Zhang, Q. F. Xiao, X. G. Zhao, Y. C. Chuang, S. R. Jin, W. M. Sun, Z. J. Li, Z. X. Zheng and H. Yang, J. Mater. Sci., 1998, 33, 1915-1919.

25 L. Li and Q. B. Wang, ACS Nano, 2013, 7, 3053-3060.

26 J. M. Lee, S. J. Kim, J. W. Kim, P. H. Kang, Y. C. Nho and Y. S. Lee, J. Ind. Eng. Chem., 2009, 15, 66-71.

27 A. C. Ferrari and J. Robertson, Philos. Trans. R. Soc., A, 2004, $362,2477$.

28 B. Lu, H. Huang, X. L. Dong and J. P. Lei, J. Phys. D: Appl. Phys., 2010, 43, 105403.

29 J. Gao, L. Zhou, J. S. Liang, Z. M. Wang, Y. Wu, J. Muhammad, X. L. Dong, H. T. Yu and X. Quan, Nano Res., 2018, 11(3), 1470-1481.

30 J. Prasek, J. Drbohlavova, J. Chomoucka, J. Hubalek, O. Jasek, V. Adamc and R. Kizek, J. Mater. Chem., 2011, 21, 15872.

31 O. V. Yazyev and A. Pasquarello, Phys. Rev. Lett., 2008, 100, 156102.

32 L. Vitos, A. V. Ruban, H. L. Skriver and J. Kollár, Surf. Sci., 1998, 411, 186.

33 S. Iijima and T. Ichihashi, Nature, 1993, 363, 603.

34 D. S. Bethune, C. H. Kiang, M. S. de Vries, G. Gorman, R. Savoy, J. Vazquez and R. Beyers, Nature, 1993, 363, 605.

35 Y. Yosida and I. Oguro, J. Appl. Phys., 1998, 83, 4985.

36 T. Skotheim, R. Elsenbaumer and J. Reynolds, Handbook of Conducting Polymers, Dekker, New York, 1998.

37 S. Iijima, Nature, 1991, 354, 56.

38 B. I. Shklovskii and A. L. Efros, Electronic properties of doped semiconductors, Springer, Berlin, 1984.

39 J. Y. Shen, Z. J. Chen, N. L. Wang, W. J. Li and L. J. Chen, Appl. Phys. Lett., 2006, 89, 153132.

40 N. Kang, J. S. Hu, W. J. Kong, L. Lu, D. L. Zhang, Z. W. Pan and S. S. Xie, Phys. Rev. B: Condens. Matter Mater. Phys., 2002, 66, 126.

41 A. Fujiwara, F. Katayama, K. Tomiyama, H. Ootoshi, H. Suematsu, M. Yumura and K. Uchida, in Proceedings of the International Winterschool on Electronic Properties of Novel Materials, "Molecular Nanostructures,", ed. H. Kuzmany, J. Fink, M. Mehring and S. Roth, World Science, Singapore, 1998. 